\title{
Anisákidos y anisakidosis: generalidades y su actualidad en Colombia. Revisión bibliográfica
}

\author{
Jenniffer Alejandra Castellanos-Garzón 1,2, Luis Felipe Falla-Zúnigaa ${ }^{3}$, Liliana Salazar, María Carolina Pustovrh-Ramos ${ }^{5}$
}

\section{RESUMEN}

La anisakidosis es una parasitosis ocasionada por nematodos de la familia Anisakidae, causada por el consumo de pescado parasitado con larvas infectivas (L3) de estos nematodos. En Europa y Asia es un problema de salud pública. Sin embargo, en Colombia y en general en los países de América del Sur, es poco conocida. El objetivo de este trabajo es presentar una revisión de las generalidades de los parásitos anisákidos y mostrar la situación actual de esta parasitosis en Colombia. Se realizó una búsqueda estructurada de términos MeSH y DeCS en MEDLINE, Cochrane, Embase, LILACS y Scopus; esta se complementó con otra no estructurada en SciELO y Google Scholar. Se incluye un reporte de caso y seis registros de hospederos. Se describen registros de peces de consumo humano parasitados por Anisakis sp., Anisakis physeterisy Pseudoterranova decipiens para el Océano Pacífico y, los géneros Pseudoterranova y Contracaecum en peces del Océano Atlántico y aguas continentales. Se concluye que la anisakidosis representa un riesgo latente para Colombia, es necesario instaurar legislaciones efectivas para un control eficiente sobre esta parasitosis y educar a la población sobre los cuidados necesarios para el consumo de pescado.

\section{PALABRAS CLAVE}

Anisakis; Colombia; Enfermedades Emerontes; Zoonosis

1 Investigadora posdoctoral. Grupo de Investigación TEBLAMI, Facultad de Salud Universidad del Valle, Cali, Colombia.

2 Profesora. Facultad de Ingeniería, Unidad Central del Valle, Tuluá, Colombia.

3 Estudiante de medicina. Grupo de Investigación TEBLAMI, Facultad de Salud Universidad del Valle, Cali, Colombia.

4 Profesora titular. Estudiante de Doctorado en Ciencias Biomédicas, Grupo de Investigación TEBLAMI, Facultad de Salud, Universidad del Valle, Cali, Colombia.

5 Profesora asociada. Jefe del Departamento de Morfología, Grupo de Investigación TEBLAMI, Facultad de Salud, Universidad del Valle, Cali, Colombia.

Correspondencia: Jenniffer Castellanos; jenniffer.castellanos@correounivalle.edu.co

Recibido: agosto 15 de 2019

Aceptado: octubre 30 de 2019

Cómo citar: Castellanos-Garzón JA, Falla-Zúñiga LF, Salazar L, Pustovrh-Ramos MC. Anisákidos y anisakidosis: generalidades y su actualidad en Colombia. Revisión bibliográfica. latreia. 2020 Abr-Jun;33(2):143-154. D0l 10.17533/udea.iatreia.47. 


\section{SUMMARY}

\section{Anisakis and Anisakiasis: General aspects and cu- rrent situation in Colombia}

Anisakidosis is a parasitic disease derived from the consumption of infective larvae from Anisakidae family. In Europe and Asia is a public health problem, however, in Colombia and South American countries is little known. The objective is to review Anikids general aspects and find out about the current situation of this disease in Colombia. Search of MeSH and DeCS terms, through MEDLINE, Cochrane, Embase, and Scopus databases, complemented with grey literature from SciELO and Google Scholar, was made. One case report and six host records were included. Records of fish for human consumption from Pacific Ocean parasitized by Anisakis sp., Anisakis physeteris and Pseudoterranova decipiens are described, as well as for fish from Atlantic Ocean and inland waters with genera Pseudoterranova and Contracaecum. Anisakidosis represents a latent risk for Colombia; it is necessary to establish effective legislations for an efficient control of the emergence of this parasitic disease, as well as to educate people about needed precautions considering fish consumption.

\section{KEY WORDS}

Anisakis; Colombia; Emerging Disease; Zoonoses

\section{INTRODUCCIÓN}

La zoonosis generada por larvas de estadio infectivo (L3) de nematodos de la familia Anisakidae se denomina anisakidosis. Esta parasitosis es ocasionada por la ingesta de pescado crudo o poco cocido parasitado con larvas (L3) ${ }^{(1)}$. El término fue introducido en 1960 por Straub y, en el mismo año, Van Thiel et al., documentaron el primer reporte de caso ${ }^{(2)}$.

La larva puede generar múltiples síntomas, aunque la mayoría pueden cursar asintomáticas ${ }^{(3)}$. Se describen tres cuadros clínicos: gastrointestinales, alérgicos y gastroalérgicos, que se explican por dos mecanismos fisiopatológicos: una reacción de hipersensibilidad inmediata y otra inflamatoria ${ }^{(4)}$.
Aunque existen casos de infestaciones múltiples, la infección suele darse por una larva ${ }^{(4)}$. Se han descrito infecciones por las especies Anisakis simplex, Anisakis physeteris, Pseudoterranova decipiens e Hysterothylacium spp. ${ }^{(2,5,6)}$. Además, algunos investigadores reportan que las manifestaciones clínicas del humano frente a los parásitos anisákidos están asociadas con el género al cual pertenece; así, los cuadros gastrointestinales se asocian con parásitos del género $P_{s e u d o-}$ terranova y los de hipersensibilidad con Anisakis ${ }^{(7,8)}$.

La mayoría de los casos se han reportado en España y Japón, en donde se presentan más de 2.000 infecciones anuales que se traducen en un problema de salud pública que ha requerido establecer legislaciones de control y prevención ${ }^{(9)}$. Sin embargo, en el 2018, Gardonete et al., ${ }^{(10)}$ reportó en Italia ocho casos de anisakidosis asociados con Anisakis sp., A. simplex (s.1.) y $A$. pegreffii como agente etiológico de la enfermedad. En Colombia y en los países de Suramérica en general, esta enfermedad es poco conocida por la población general y el personal de salud. La información reportada para anisákidos en la región corresponde en su mayoría a tesis de pregrado y posogrado ${ }^{(11)}$. Este desconocimiento puede tener como consecuencia un subdiagnóstico de la enfermedad. El objetivo de este trabajo es presentar una revisión descriptiva de la anisakidosis desde los aspectos generales del parásito, los reportes de caso y los hospederos identificados que probablemente estén involucrados en su transmisión en Colombia.

\section{MÉTODOS}

Se realizó una revisión de la literatura para identificar los aspectos básicos de los nematodos anisákidos y la información reportada para Colombia: reportes de casos clínicos y las especies de peces registradas como hospederos.

La búsqueda se realizó en cada base de datos consultada hasta mayo del 2019. Se revisaron las bases de datos MEDLINE, Cochrane, Embase, LILACS y Scopus. Se utilizaron los términos MeSH y DeCS: anisákidos, anisakiasis, anisakidosis, Anisakidae, Anisakis, Pseudoterranova y Contracaecum, seguido de la conjunción “AND” y la palabra Colombia. 
Como criterios de inclusión, se incorporaron todos los artículos publicados en inglés y español sobre casos de humanos reportados de Colombia con evidencia de larvas L3 de anisákidos. Para los reportes de los hospederos se incluyeron los estudios con peces parasitados por la familia Anisakidae, tanto de aguas continentales como marítimas. Se excluyeron los estudios donde la información para determinar la elegibilidad no estaba disponible.

Se complementó con una búsqueda no estructurada en SciELO y Googile Scholar. Además, se consultó con la directora del grupo de investigación TEBLAMI, de la Universidad del Valle quienes han realizado importantes aportes sobre anisákidos, para identificar referencias adicionales como tesis de preogrado y posorado con resultados inéditos.

\section{RESULTADOS}

El ciclo de vida del parásito inicia con la expulsión de huevos a través de las heces de mamíferos marinos que son sus hospederos definitivos. En el agua, favorecidos por temperaturas entre 13 y $18^{\circ} \mathrm{C}(12,13)$, Ios huevos embrionan, eclosionan y liberaran larvas del primer estadio (L1) que, posteriormente, mudan a un segundo estadio larval (L2) ${ }^{(14)}$. Las larvas L2 son ingeridas por crustáceos pequeños ${ }^{(15)}$, que a su vez son consumidos por peces, hospederos intermediarios, donde mudan al tercer estadio larval (L3). Finalmente, los peces parasitados con L3 son ingeridos por mamíferos marinos, sus hospederos definitivos, donde pasan a L4 y a su forma adulta; se reproducen $y$ liberan huevos, iniciando un nuevo ciclo ${ }^{(16)}$.

Se ha encontrado que el hospedero definitivo (mamífero marino), en el caso del género Pseudoterranova, está asociado con los pinnípedos (focas, leones marinos, morsas, etc.); mientras que, Anisakis completa el ciclo en cetáceos (ballenas y delfines) ${ }^{(16)}$. Además, se han hallado aves también como hospederos definitivos de anisákidos del género Contracaecum ${ }^{(17)}$. En el ciclo de vida en general participan múltiples hospederos intermediarios, dependiendo de la zona geográfica en donde se desarrolle ${ }^{(18)}$.

Cuando un pez parasitado muere, la larva infectante migra desde las vísceras hasta el músculo ${ }^{(19)}$. EI humano que ingiere la carne cruda o semicruda del pescado parasitado con larvas L3 puede resultar infectado, pasando a ser un hospedero accidental donde no se desarrolla el parásito adulto ${ }^{(20)}$. Estos parásitos ocasionan la enfermedad anisakidosis en el ser humano, la cual se asocia con cuadros gástricos, alérgicos y gastroalérgicos ${ }^{(21)}$.

\section{Taxonomía}

Los nematodos ascaroideos de la familia Anisakidae (Nematoda) están comprendidos por 24 géneros, los más conocidos Pseudoterranova, Contracaecum y Anisakis ${ }^{(22)}$. En la actualidad, están descritas nueve especies de Anisakis ${ }^{(22.23-25)}$, siendo A. simplex el principal responsable de los episodios aléroicos ${ }^{(26)}$. Se estima que Pseudoterranova y Contracaecum son de menor importancia, aunque desde el punto de vista epidemiológico es necesario evaluar su incidencia en la población, especialmente lo asociado con la sensibilización a los alérgenos ${ }^{(27)}$.

\section{Morfología}

Los anisákidos tienen un cuerpo vermiforme, no segmentado. Su extremo posterior termina en forma cónica o puntiaguda ${ }^{(13)}$. Poseen una cutícula de 1,50 $\mu \mathrm{m}$ de grosor ${ }^{(12)}$, que en algunas especies presenta estriaciones finas transversales más marcadas hacia la parte posterior del cuerpo ${ }^{(28)}$. El sistema digestivo es completo. El esófago mide de 2,5 a 3,0 mm, con una porción anterior muscular, el proventrículo y una posterior glandular, el ventrículo ${ }^{(29)}$. Poseen un intestino que ocupa la mayor parte de la longitud del nematodo, su extremo posterior es redondeado y termina en un mucrón o espina. Tienen un anillo nervioso en el tercio anterior de su cuerpo ${ }^{(30)}$.

EI género Anisakis (Figura 1A) posee un ventrículo Iarogo con una unión ventrículo-intestinal oblicua, cola redondeada, corta con una espina terminal ${ }^{(12)}$. EI género Pseudoterranova (Figura 1B) presenta ciego intestinal bien desarrollado, se extiende hacia el extremo anterior, sobrepasa el margen anterior del ventrículo y es reconocido por la ausencia de apéndice esofágico. Presenta una cola postanal corta y cóni$\mathrm{ca}$, con una espina terminal fina. El extremo anterior tiene un diente pequeño $y$ triangular dirigido hacia afuera. El poro excretor aparece situado justo en posición ventral al diente ${ }^{(12)}$. 


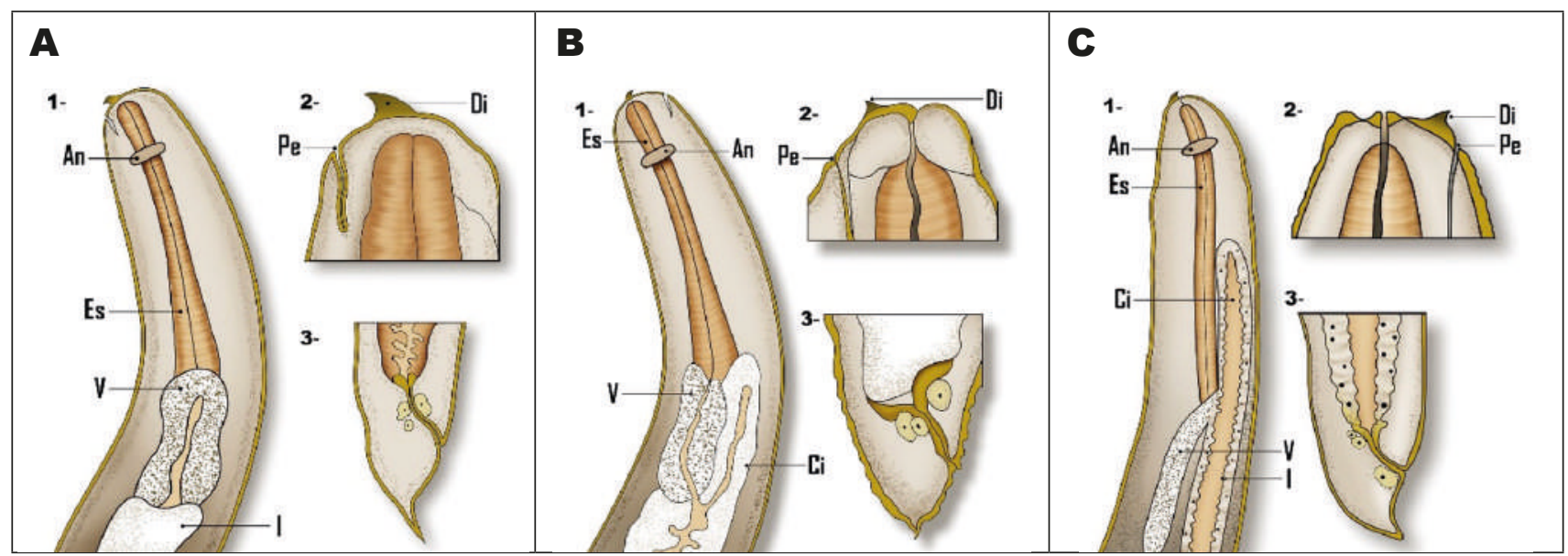

Figura 1. Características morfológicas de las larvas en L3 de A. Anisakis. B. Pseudoterranova. C. Contracaecum. 1: parte anterior; 2: extremo anterior; 3: extremo posterior; An: anillo nervioso; Es: esófago; V: ventrículo; I: intestino; Di: diente cuticular; Pe: poro excretor. Fuente: creación propia

Las larvas de Contracaecum (Figura $1 \mathrm{C}$ ) miden entre 4 y $16 \mathrm{~mm}$. En el extremo anterior posee tres labios, un diente cuticular cónico y ligeramente romo. El poro excretor es continuo al diente. En el tubo digestivo poseen ventrículo pequeño y esférico, apénđice ventricular y un ciego anterior más pequeño que el apéndice ventricular. El extremo posterior es cónico, la cola postanal larga y no presenta mucrón ${ }^{(12)}$.

\section{Anisakidosis}

Es la infección causada por los nematodos de la familia Anisakidae. Sin embargo, cuando esta es causada por algunas especies del género Anisakis, se denomina anisakiasis. Este cuadro clínico es frecuente en Asia y Europa asociado con reacciones alérgicas, mientras la causada por Pseudoterranova es conocida como pseudoterranovosis, siendo este el género responsable de la mayoría de casos reportados en el continente americano, principalmente, en Chile ${ }^{(2,23,31-34)}$ y Perú ${ }^{(20,35,36)}$. Existe un reporte de infección por $C$. osculatum en Japón y cuatro reportes por H. aduncum: dos en Corea del Sur y dos en Japón; no se conocen reportes en América del Sur hasta el momento ${ }^{(37)}$.

En cuanto a las formas de presentación de la anisakidosis, tenemos que, la parasitación en el tracto digestivo humano por las larvas L3 es la de mayor prevalencia. Como formas gastrointestinales se pueden encontrar: 1. La forma luminal no invasiva, en la cual
Ia larva puede ser eliminada a través de la materia fecal o el vómito; 2. La forma gástrica, cuando la larva invade la mucosa gástrica y requiere endoscopia ${ }^{(2,4)}$; 3. Forma intestinal, cuando la larva migra al intestino delogado, cursa con síntomas como el dolor abdominal, diarrea, fiebre, entre otros ${ }^{(2)} ; y, 4$. Forma gastrointestinal, cuando las larvas alcanzan la cavidad abdominal y pueden migrar al pulmón, el páncreas e hígado ${ }^{(33)}$.

La sintomatología gastrointestinal depende de la ubicación. Según los reportes, la localización esofágica se presenta en el 0,8\% de los casos ${ }^{(33)}$; la gástrica, en la mayoría $70-95 \%{ }^{(2,4)} y$, la intestinal, con un $4 \%$ de ellos ${ }^{(2)}$ con una variante ileal terminal y una colónica rara, en el 0,1-0,9\% ${ }^{(38)}$. Existen reportes en los cuales la larva se ubicó en la laringe y lengua ${ }^{(7)}$ o migró al hígado, bazo, páncreas, los pulmones, ovarios o ganglios linfáticos ${ }^{(2,7)}$.

Los cuadros gastrointestinales varían en sintomatología de acuerdo con la presentación y evolución cronológica de cada caso. Sin embaroo, la mayoría son autolimitados ${ }^{(4)}$. Cuando la afección es más proximal los síntomas inician tempranamente, 6 a 12 horas después de la ingestión del pescado parasitado; la larva puede desencadenar el reflejo tusígeno que permite la expulsión del parásito con remoción manual ${ }^{(31)}$. Pueden presentarse, además, dolor faríngeo, prurito nasal, náusea y vómito ${ }^{(7,8)}$. 
En los cuadros gástricos, la larva se ubica en los pliegues de la curvatura mayor del estómago, pasando desapercibida en el examen endoscópico ${ }^{(39)}$; los pacientes cursan con un dolor epigástrico intenso (pirosis) y repentino, inicia de 12 a 72 horas posteriores a la comida y no mejora con analósésicos ${ }^{(2,4)}$. Asimismo, se puede

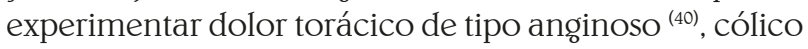
abdominal, náuseas, sensación de estómago vacío ${ }^{(41)}$, febrícula y distensión abdominal por ascitis ${ }^{(39)}$.

Cuando está comprometido el intestino, los síntomas aparecen tardíamente, incluso semanas después de la infección. En estos casos prevalece la obstrucción intestinal, seguida por una perforación en el intestino que se puede complicar en peritonitis, heces melénicas, dilatación proximal del intestino delgado ${ }^{(7,39)} \mathrm{O}$ intususcepción ${ }^{(38)}$. Ocasionalmente, la larva puede ser eliminada en las deposiciones o migrar hasta el ano ${ }^{(2.32)}$.

La infección también puede desencadenar respuestas aléroicas agudas, estas abarcan urticarias, angioedema y shock anafiláctico. Suelen aparecer entre 4 a 24 horas después de la ingesta ${ }^{(2)}$. El sistema inmune reconoce los antígenos excretados/secretados de la larva viva y los de la cutícula en la larva muerta ${ }^{(8)}$. Hasta la fecha, se tienen identificados trece alérgenos de $A$. simplex;

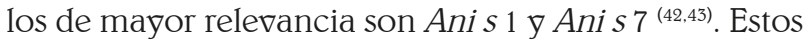
alérgenos desencadenan una reacción de hipersensibilidad tipo I, mediada por IogE típica de un perfil linfocítico Th2 $2^{(39)}$, la cual requiere de una sensibilización previa de las células del sistema inmune, probablemente, por un cuadro intestinal asintomático anterior ${ }^{(3,44)}$.

La reacción inmunológica en el humano puede ser aguda o crónica y está determinada por el tiempo de permanencia de la larva ${ }^{(20)}$. El tipo agudo se caracteriza por eritema e hiperemia en la mucosa ${ }^{(41)}$, acompañado de edema que puede comprometer la submucosa (39). Al aumentar la permanencia de la larva varios días, esta puede migrar hasta la submucosa y generar alteraciones de tipo crónico, con infiltración eosinofílica ${ }^{(4)}$ que termina en la formación de abscesos y granulomas por una reacción de hipersensibilidad tipo IV (7,8).

La larva muere de 8 a 24 semanas después de ser ingerida ${ }^{(2,7)}$. En cuanto a las reacciones alérgicas crónicas, la urticaria es una manifestación común, también se ha asociado con rinitis, dermatitis, conjuntivitis y asma ocupacional (en pescadores) e infantil ${ }^{(2,7,20,45)}$. Se debe aclarar que ambas presentaciones pueden ocurrir en conjunto como un cuadro gastroaléroico ${ }^{(40)}$.
Debido a la naturaleza termoestable de los antígenos como Ani s 4 de $A$. simplex, se pueden presentar reacciones alérgicas aún con el consumo de pescado bien cocido, situación que constituye un problema para el $27 \%$ de los individuos sensibilizados ${ }^{(21,45)}$.

La identificación de genes que codifican para proteínas con un papel antigénico en los productos de excreción y secreción, ha permitido identificar A.peg-1, una glucoproteína: A.peg-7 y la mioglobina A.peg-13 como posibles proteínas desencadenantes de la respuesta alérgica ${ }^{(46)}$.

Para el diagnóstico de anisakidosis se tiene en cuenta el cuadro clínico del paciente y el antecedente de consumo de pescado. El diagnóstico de un cuadro gastrointestinal exige la identificación de la larva, ya sea in vivo, por medio de endoscopia o, in situ, en una biopsia. La endoscopia temprana es el gold standard para el diaognóstico ${ }^{(4)}$.

Por la similitud en la presentación clínica, se debe hacer un diagnóstico diferencial de los cuadros gastrointestinales como la gastritis, apendicitis aoguda, pseudoobstrucción intestinal u otras infecciones parasitarias, infecciones bacterianas, enfermedad de Crohn y neoplasias, principalmente, en los casos crónicos con formación de granulomas que simulan tumores ${ }^{(4,7,8,36)}$.

Para los cuadros alérogicos no es indispensable la observación directa de la larva, ya que los síntomas se atribuyen principalmente a la respuesta inmune del hospedero, por ello se requieren pruebas serológicas. La detección de anticuerpos IogM, IogG e IogE, específicos contra el parásito, son un método sensible ${ }^{(3)} \mathrm{O}$, las pruebas cutáneas como el test de Prick, indican un contacto previo con el nematodo y se usan en investigación ${ }^{(42)}$.

En casos clínicos asociados con larva viva, el tratamiento consiste en la extracción por endoscopia con fórceps, realizando una remoción completa del nematodo para su identificación. Los síntomas suelen desaparecer pocas horas después de la extracción ${ }^{(41)}$.

En casos con granulomas u obstrucciones intestinales, se debe hacer una resección de los segmentos afectados (7). Se han propuesto tratamientos empíricos con antiparasitarios como el albendazol y mebendazol, que buscan erradicar la larva, pero no se conoce su efectividad ${ }^{(39,44)}$.

En las reacciones aléroicas el tratamiento inmediato abarca antihistamínicos, broncodilatadores y vasoconstrictores en los casos más graves ${ }^{(44)}$. 


\section{Anisakidosis en el mundo}

Las nematodiasis transmitidas por el consumo de alimentos es actualmente un problema de salud pública para países de la Unión Europea ${ }^{\left({ }^{47}\right)}$. La mayoría de los casos están asociados con el consumo de productos crudos o poco cocidos, con la falta de higiene en el procesamiento de alimentos, provocando así enfermedades que pueden ser evitadas. Entre ellas, la más prevalente, asociada con la ingesta de pescado es la anisakidosis, considera un problema de salud pública en Europa y Asia, donde la enfermedad es conocida, diagnosticada y cuenta con una legislación clara para minimizar la aparición de ella. Entre los años 2000 y 2016 se reportaron en Europa 236 casos (únicamente en la literatura científica), todos ellos autóctonos y de diagnóstico definitivo ${ }^{(47)}$.

En 62 pacientes, los autores especificaron explícitamente que la infección fue producida por la ingestión de anchoa (Enoraulis encrasicolus); España es el país con el mayor número de casos reportados: $158^{(48)}$, el segundo fue Italia con $67^{(10)}$. Como método de diagnóstico es común realizar serología en más del $87 \%$ de los casos. Sin embargo, en el 3 \% de los reportes el diagnóstico fue realizado por laparotomía exploratoria o gastroscopia ${ }^{(47)}$.

\section{Perspectiva en Colombia}

La ubicación geográfica de Colombia con dos océanos y su relación con hábitats marinos y países como Ecuador, donde han sido reportados peces de consumo humano parasitados por nematodos de la familia Anisakidae ${ }^{(49.50)}$, hacen que la pesca represente no solo una actividad económica importante, sino un factor de riesogo para la infección si esta no es manejada adecuadamente.

En la revisión, se encontraron 17 especies reportadas como hospederos, tanto en peces de agua marítima como continental. En el Océano Pacífico se ha reportado en los puertos de Buenaventura y Tumaco y, en el Atlántico, en Cartagena y la Ciénaga del Totumo (49,51,52). De aguas continentales, los ríos Amazonas, Atrato, Cauca, Magdalena, San Joroge y Sinú ${ }^{(51,53,54)}$.

Se han reportado peces de consumo humano parasitados por Anisakis sp., A. physeteris y P. decipiens en el Océano Pacífico. Los géneros Pseudoterranova y Contracaecum en peces del Atlántico y, Contracaecum, en especies de aguas continentales ${ }^{(10,11,45,51)}$

En la Tabla 1 se presenta el listado de peces reportados en Colombia como hospederos de nematodos anisákidos y, en la Figura 2, el mapa de su distribución.

Tabla 1. Especies de peces reportados como hospederos intermediarios de parásitos de la familia Anisakidae en Colombia

\begin{tabular}{|c|c|c|c|c|c|}
\hline \multicolumn{6}{|c|}{ Hospedero (pez) } \\
\hline Nombre científico & Nombre común & Lugar del estudio & Nematodo identificado & $\begin{array}{l}\text { Peces } \\
\text { revisados } \\
\text { (n) }\end{array}$ & $\begin{array}{l}\text { Prevalencia } \\
\text { de la infección } \\
\text { (\%) }\end{array}$ \\
\hline Ageneiosus caucanus & Doncella & San Marcos, Sucre & Contracaecum sp. & 60 & 23,3 \\
\hline Caquetaia kraussii & Mojarra amarilla & San Marcos, Sucre & Contracaecum sp. & 60 & 95 \\
\hline Caranx hippos & Jurel & Cartagena, Bolívar & Familia Anisakidae & 12 & 8,5 \\
\hline Centropomus armatus & Róbalo & Buenaventura, Valle del Cauca & Anisakis physeteris & 12 & 42 \\
\hline $\begin{array}{l}\text { Centropomus } \\
\text { undecimalis }\end{array}$ & Róbalo & Cartagena, Bolívar & Familia Anisakidae & 23 & 4,3 \\
\hline \multirow{3}{*}{ Hoplias malabaricus } & \multirow{3}{*}{ Moncholo } & Cartagena, Bolívar & Contracaecum sp. & 227 & 100 \\
\hline & & San Marcos, Sucre & Contracaecum sp. & 60 & 93,3 \\
\hline & & Rio Sinú y San Jorge, Córdoba & Contracaecum spp. & 45 & 100 \\
\hline Larimus argenteus & Silver drum & Buenaventura, Valle del Cauca & Anisakis physeteris & 2 & 100 \\
\hline Merluccius gayi & Merluza & $\begin{array}{l}\text { Cartagena, Bolívar } \\
\text { y Barranquilla, Atlántico }\end{array}$ & Familia Anisakidae & 134 & 24,6 \\
\hline \multirow{3}{*}{ Mugil cephalus } & \multirow{2}{*}{ Lisa } & Buenaventura, Valle del Cauca & Anisakis physeteris & 12 & 33 \\
\hline & & Buenaventura, Valle del Cauca & Anisakis sp. & 15 & 33 \\
\hline & Lebranche & Cartagena, Bolívar & Familia Anisakidae & 19 & 100 \\
\hline
\end{tabular}


Tabla 1. Especies de peces reportados como hospederos intermediarios de parásitos de la familia Anisakidae en Colombia (Continuación)

\begin{tabular}{|c|c|c|c|c|c|}
\hline \multicolumn{6}{|c|}{ Hospedero (pez) } \\
\hline Nombre científico & Nombre común & Lugar del estudio & Nematodo identificado & $\begin{array}{l}\text { Peces } \\
\text { revisados } \\
\text { (n) }\end{array}$ & $\begin{array}{l}\text { Prevalencia } \\
\text { de la infección } \\
\text { (\%) }\end{array}$ \\
\hline \multirow{2}{*}{ Mugil curema } & \multirow{2}{*}{ Lisa } & Tumaco, Nariño & Anisakis physeteris & 16 & 94 \\
\hline & & Tumaco, Nariño & Pseudoterranova decipiens & 16 & 94 \\
\hline \multirow{3}{*}{ Mugil incilis } & \multirow{3}{*}{ Lisa } & Cartagena, Bolívar & Contracaecum spp. & 378 & 100 \\
\hline & & Cartagena, Bolívar & Pseudoterranova spp. & 378 & 80,5 \\
\hline & & Cartagena, Bolívar & Familia Anisakidae & 355 & 83,9 \\
\hline Mugilspp. & Múgil & Cartagena, Bolívar & Familia Anisakidae & 5 & 100 \\
\hline Plagioscion magdalenae & Pacora & San Marcos, Sucre & Contracaecum sp. & 60 & 46,7 \\
\hline $\begin{array}{l}\text { Pseudoplatystoma } \\
\text { magdaleniatum }\end{array}$ & Bagre pintado & San Marcos, Sucre & Contracaecum sp. & 60 & 95 \\
\hline Salminus affinis & Rubio & Rio Sinú y San Jorge, Córdoba & Contracaecum sp. & 45 & 95 \\
\hline Sciades herzbergii & Barbul chivo & Cartagena, Bolívar & Familia Anisakidae & 64 & 1,6 \\
\hline Sorubim cuspicaudus & Blanquillo & San Marcos, Sucre & Contracaecum sp. & 60 & 100 \\
\hline
\end{tabular}

Fuente: creación propia, basada en los datos obtenidos a partir de las referencias citadas (11,49,51-55)

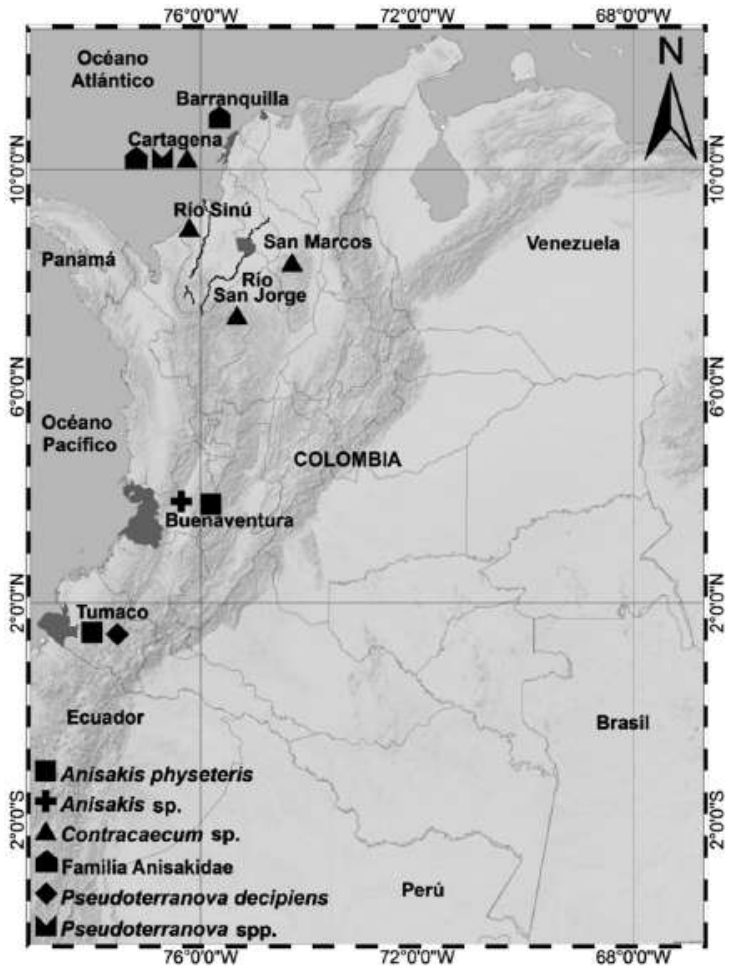

Figura 2. Distribución de nematodos anisákidos reportados en CoIombia como hospederos intermediarios de peces para el consumo humano. Fuente: adaptación referencias ${ }^{(11,49,51-55)}$
Como se observa en la Tabla 1, los peces del grupo de los mugílidos, conocidos como "lisas", de los cuales las especies Mugil cephalus, $M$. incilis y $M$. curema, existen en Colombia, son los que se reportaron con mayor frecuencia como hospederos intermediarios de nematodos anisákidos tipo Anisakis sp., A. physeteris, P. decipiens y Contracaecum sp. ${ }^{(11,49,51,52,55,56)}$. Los porcentajes de infección en los lisas varía entre el 33 al 100 \% ${ }^{(11,49,51-56)}$, este alto porcentaje podría deberse a que estas especies de peces se encuentran tanto en aguas de la Costa Atlántica como en la Pacífica, siendo especies de importancia económica y comercial ${ }^{(10)}$.

En general, los porcentajes de infección por especie de pez se reportan entre un 1,4 a un $100 \%^{(11,49,51-56)}$.

En los estudios realizados en Cartagena, Ciénaga del Totumo y Barranquilla, Ias especies de peces de consumo Caranx hipos, Centropomus undecimalis, MerIuccius gayi, Mugil cephalus, Mugil incilis, Sciades herzbergii, se reportaron como hospederos de nematodos anisákidos ${ }^{(51,55,56)}$. En estos estudios la determinación taxonómica se logró hasta el nivel de familia $(51,55,56)$, probablemente por la complejidad morfológica del estadio larval para su identificación. Por lo tanto, es importante realizar investigaciones que ayuden a la identificación al nivel de la especie, pues existen diferencias clínicas en las manifestaciones de la enfermedad producida por cada una. 
En el Pacífico, los reportes indican que los géneros Anisakis ${ }^{(11)}$, Pseudoterranova ${ }^{(52)}$, Contracaecum ${ }^{(51-54)}$ y, las especies A. physeteris ${ }^{(49)}$ y $P$. decipiens, parasitaron a los peces de consumo Centropomus armatus y Mugil curema, respectivamente, comercializados en Buenaventura y Tumaco ${ }^{(49)}$.

Los reportes de la presencia de Contracaecum sp., están relacionados con peces de aguas continentales. A la fecha no se conocen registros de este género en la región del Pacífico colombiano. Es importante recalcar que el género Contracaecum no cuenta con registros de parasitosis asociadas con humanos y, además, presenta una variación al ciclo de vida, donde este puede involucrar un ave como hospedero intermediario, evento que explica su presencia en peces de agua dulce ${ }^{(47.51)}$

En la bibliografía consultada solo se encontró un reporte de caso de anisakidosis en Colombia, presentado por Patiño y Olivera (2019). Se definió como de tipo gástrico en una mujer de 52 años, quien posterior a la ingesta de comida típica a base de pescado crudo, desarrolló un cuadro clínico de dos días, consistente en epigastralgia grave, asociada con hiporexia, náuseas, emesis, diarrea y urticaria.

Por hallazogos de dolor a la palpación en la región periumbilical, leucocitosis a expensas de neutrófilos y eosinofilia, se realizó una endoscopia digestiva alta donde se observó la larva alojada en la mucosa antral. Esta fue extraída y fijada en glutaraldehído para un posterior análisis patológico en el que se describió una larva de Anisakis simplex (sensu lato). La paciente presentó remisión de síntomas ulteriores a la extracción del parásito. Además, se le inició manejo con albendazol $400 \mathrm{mg}$, dosis única ${ }^{(57)}$.

Resaltamos que este reporte fue realizado después de las recientes publicaciones realizadas por Castellanos JA. et al. (2018), donde se describe, por primera vez para Colombia, la presencia de $A$. physeteris y $P$ decipiens en peces de consumo comercializados en el puerto de Buenaventura y se hace un llamado a la comunidad científica, el personal de salud y la comunidad en general sobre el riesgo en Colombia de la anisakidosis como una posible enfermedad emergente ${ }^{(49)}$.

En cuanto a la anisakiasis alérogica ${ }^{(3,58,59)}$ en Colombia, se conoce una tesis doctoral donde se documenta en una población de Cali la presencia de anticuerpos dirigidos contra antígenos específicos de A. simplex. La investigación reportó que la población de estudio consumía pescado tres veces al mes y, de un análisis de 150 sueros, se encontró que el 1,3\% de la población presento anticuerpos específicos frente al antígeno recombinante Ani $s 7$ de $A$. simplex, siendo este reconocido por el $100 \%$ de las personas que han estado en contacto con el parásito ${ }^{(60)}$.

\section{CONCLUSIONES}

Esta revisión permite conocer el panorama actual de la anisakidosis en Colombia respecto a los casos clínicos reportados en humanos, $y$ frente a las especies de peces que se han identificado como hospedadores intermediarios de los nematodos anisákidos en Colombia y demás países vecinos; evidencias que llevan a pensar en esta parasitosis como una enfermedad emergente, zoonosis que por desconocimiento del personal de salud puede estar subdiagnosticada.

Asociado a lo anterior, el aumento de la oferta de preparaciones a base de pescado y mariscos crudos o poco cocidos de origen mediterráneo u oriental, como el sushi y cebiche, obliga a hacer un llamado de atención a las autoridades de salud, con el fin de reforzar la vigiilancia de las buenas prácticas de manufactura y elaboración de alimentos, con el objetivo de contrarrestar los peligros que puedan deteriorar la calidad e inocuidad de alimentos derivados del pescado.

En este sentido, consideramos que se requieren estrategias de promoción y prevención, educación médica, implementación de una legislación sobre el manejo del pescado: desde la evisceración temprana posterior a la captura de los peces hasta los procesos de almacenamiento, que requieren congelación a temperaturas estándar $\left(-20^{\circ} \mathrm{C}\right)$, todo esto para tener un control en el número de peces infectados, prevenir el número de casos y combatir el subregistro. No sobra decir que esta medida fue implementada en los Países Bajos donde fue evidente la reducción de los casos.

Finalmente, en Colombia solo se ha reportado un caso de anisakidosis, lo que pone de manifiesto la necesidad de ampliar el estudio de esta parasitosis que se presenta con síntomas poco específicos y diversos que dificultan el diagnóstico. 


\section{AGRADECIMIENTOS}

A Colciencias por la Convocatoria Nacional 567 para estudios de Doctorado en Colombia.

\section{CONFLICTOS DE INTERESES}

Ninguno por declarar.

\section{FINANCIACIÓN}

Ninguno declarado por los autores.

\section{REFERENCIAS BIBLIOGRÁFICAS}

1. Hochberg NS, Hamer DH. Anisakidosis: Perils of the deep. Clin Infect Dis. 2010;51(7):806-12. DOI $10.1086 / 656238$.

2. Jofré ML, Neira OP, Noemí HI, Cerva CJL. Pseudoterranovosis and sushi. Rev Chilena Infectol. 2008;25(3):2005. DOI 10.4067/S0716-10182008000300010.

3. Figueiredo Junior I, Vericimo MA, Cardoso LR, São Clemente SC, do Nascimento ER, Teixeira GA. Crosssectional study of serum reactivity to Anisakis simplex in healthy adults in Niterói, Brazil. Acta Parasitol. 2013 Sep;58(3):399-404. DOI 10.2478/s1 1686-013-0157-3.

4. Peláez MA, Codoceo CM, Montiel PM, Gómez FS, Castellano G, Herruzo JAS. Anisakiasis múltiple. Rev Esp Enferm Dig. 2008;100(9):581-2. DOI 10.4321/ s1130-01082008000900009.

5. Cabrera R. Anisakiasis outbreak by Anisakis simplex larvae associated to Peruvian food in Spain. Rev Esp Enfermedades Dig. 2010;102(10):610-1. DOI 10.4321/ S1130-01082010001000011.

6. Picó-Durán G, Pulleiro-Potel L, Abollo E, Pascual S, Muñoz P. Molecular identification of Anisakis and Hysterothylacium larvae in commercial cephalopods from the Spanish Mediterranean coast. Vet Parasitol. 2016 Apr;220:47-53. DOI 10.1016/j.vetpar.2016.02.020.

7. Nieuwenhuizen N. Anisakis - Immunology of a foodborne parasitosis. Parasite Immunol. 2016;38(9):54857. DOI 10.1111/pim.12349.

8. Terán-Angel G, Rojas J. Anisakidosis, inflamación e hipersensibilidad (Anisakidosis, inflammation and hypersensitivity). Avan Biomed. 2012;1(1):30-7.
9. Audícana MT, Ansotegui IJ, Fernández de Corres L, Kennedy MW. Anisakis simplex: dangerous--dead and alive? Trends Parasitol. 2002;18(1):20-5. DOI 10.1016/ S1471-4922(01)02152-3.

10. Guardone L, Armani A, Nucera D, Costanzo F, Mattiucci S, Bruschi F. Human anisakiasis in Italy: A retrospective epidemiological study over two decades. Parasite. 2018;25(41):1-21. DOI 10.1051/parasite/2018034.

11. Castellanos JA, Tangua AR, Salazar L. Anisakidae nematodes isolated from the flathead grey mullet fish (Mugil cephalus) of Buenaventura, Colombia. Int J Parasitol Parasites Wildl. 2017;6(3):265-70. DOI 10.1016/j.ijppaw.2017.08.001.

12. Olivero J, Baldiris R. Parásitos en peces colombianos: ¿están enfermando nuestros ecosistemas? [internet]. [Consultado 2018 abr 13]. Disponible en: https://n9.cl/ fsc $6 y$

13. Smith JW, Wootten R. Anisakis and Anisakiasis. Adø Parasitol. 1978;16:93-163. DOI 10.1016/S0065-308X(08)60573-4.

14. Ksie M, Berland B, Burt MDB. Development to thirdstage larvae occurs in the eggs of Anisakis simplex and Pseudoterranova decipiens (Nematoda, Ascaridoidea, Anisakidae). Can J Fish Aquat Sci. 1995;52:134-9. DOI 10.1139/f95-519.

15. Wilhelm Busch M, Kuhn T, Munster J, Klimpel S. Marine Crustaceans as Potencial Hosts and Vectors for Metazoan Parasites. In: Mehlhorn Heinz, editor. Parasitology Research Monographs. 3 ed. Heidelberg: Springer; 2012. p. 332-40.

16. KlimpeI S, PaIm HW. Anisakid Nematode (Ascaridoidea ) Life Cycles and Distribution : Increasing Zoonotic Potential in the Time of Climate Change? In: Mehlhorn H, editor. Berlin, Heidelberg: Springer; 2011. p. 201-22.

17. Mattiucci S, Paoletti M, Olivero-Verbel J, Baldiris R, Arroyo-Salgado B, Garbin L, et al. Contracaecum bioccai n. sp. from the brown pelican Pelecanus occidentalis (L.) in Colombia (Nematoda: Anisakidae): morphology, molecular evidence and its genetic relationship with congeners from fish-eating birds. Syst Parasitol. 2008;69(2):101-21. DOI 10.1007/s1 1230-007-9116-4.

18. McClelland G. The trouble with sealworms (Pseudoterranova decipiens species complex, Nematoda): a review. Parasitology. 2002;124 SuppI:S183-203. DOI $10.1017 / \mathrm{s} 0031182002001658$.

19. Tuemmers C, Nuñez C, Willgert K, Serri M. Anisakiasis y Difilobotriasis. Ictiozoonosis de riesgo para la salud 
pública asociada al consumo del pescado crudo en Chile. Rev la Unir del Zulia. 2014;5(11):27-39.

20. Cabrera R, Trillo-Altamirano MP. Anisakidosis: ¿una zoonosis parasitaria marina desconocida o emergente en el Perú? Rev. gastroenterol. Perú. 2004;24:335-42.

21. Torres P, Puga S, Castillo L, Lamilla J, Miranda JC. HeIminths, myxozoans and microsporidians in muscles of commercialised fresh fish and their importance as potential risk for human health in the city of Valdivia, Chile. Arch Med Vet. 2014;46(1):83-92. DOI 10.4067/ S0301-732X2014000100012.

22. Mattiucci S, Nascetti G. Chapter 2 Advances and Trends in the Molecular Systematics of Anisakid Nematodes, with Implications for their Evolutionary Ecology and Host-Parasite Co-evolutionary Processes. Adv Parasitol. 2008;66(08):47-148. DOI 10.1016/ S0065-308X(08)00202-9.

23. Mattiucci S, Pagoi L, Nascetti G, Portes Santos C, Costa G, Di Beneditto AP, et al. Genetic markers in the study of Anisakis typica (Diesing, 1860): Larval identification and genetic relationships with other species of Anisakis Dujardin, 1845 (Nematoda: Anisakidae). Syst Parasitol. 2002;51(3):159-70. DOI 10.1023/A: 1014554900808.

24. Mattiucci S, Paggi L, Nascetti G, Abollo E, Webb SC, pascual S, et al. Genetic divergence and reproductive isolation between Anisakis brevispiculata and Anisakis physeteris (Nematoda: Anisakidae). Int J. Parasitol. 2001;31(1):9-14. DOI 10.1016/S0020-7519(00)00125-9.

25. Mattiucci S, Cimmaruta R, Cipriani P, Abaunza P, BeIlisario B, Nascetti G. Integrating Anisakis spp. parasites data and host genetic structure in the frame of a holistic approach for stock identification of selected Mediterranean Sea fish species. Parasitology. 2015;142:90-108. DOI 10.1017/S0031182014001103.

26. Audicana MT, Kennedy MW. Anisakis simplex: From obscure infectious worm to inducer of immune hypersensitivity. Clin Microbiol Rev. 2008;21(2):360-79. DOI 10.1128/CMR.00012-07.

27. Fitzsimmons CM, Falcone FH, Dunne DW. Helminth allergens, parasite-specific IgE, and its protective role in human immunity. Front Immunol. 2014:1-12. DOI 10.3389/fimmu.2014.00061.

28. Fukuda T, Aji T, Tonga Y. Surface ultrastructure of larval Anisakidae (Nematoda: Ascaridoidea) and its identification by mensuration. Acta Med Okayama. 1988;42(105-16). DOI 10.18926/AMO/31010.
29. Osanz Mur AC. Presencia de larvas de anisákidos (Nematoda: Ascaridoidea) en pescado de consumo capturado en la zona pesquera de Tarragona. [Memoria doctorado en Veterinaria]. Barcelona: Universidad Autonoma de Barcelona; 2002.

30. Baird FJ, Gasser RB, Jabbar A, Lopata AL. Foodborne anisakiasis and allergy. Mol Cell probes. 2014;28(4):167-74. DOI 10.1016/j.mcp.2014.02.003.

31. Mercado R, Torres P, Muñoz V, Apt W. Human infection by Pseudoterranova decipiens (Nematoda, Anisakidae) in Chile: report of seven cases. Mem Inst Oswaldo Cruz. 2001 Jul;96(5):653-5. DOI 10.1590/ S0074-02762001000500010.

32. Torres P, Jercic MI, Weitz JC, Dobrew EK, Mercado RA. Human Pseudoterranovosis, an Emerging Infection in Chile. J Parasitol. 2007;93(2):440-3. DOI 10.1645/ GE-946R.1.

33. Mercado PR, Torres HP, Gil LC, Goldin GL. Anisakiasis en un paciente portadora de una pequeña hernia hiatal. Caso clínico. Rev Med Chil. 2006;134(12):15624. DOI 10.4067/S0034-98872006001200011.

34. Weitzel T, Sugiyama H, Yamasaki H, Ramirez C, Rosas R, Mercado R. Human infections with Pseudoterranova cattani nematodes, Chile. Emerg Infect Dis. 2015;21(10):1874-5. DOI 10.3201/eid2110.141848.

35. Tanteleán M, Huiza A. Larva de nemátodos con importancia médica hallados en peces del mar de Perú y dos primeros casos de infección humana. Rev Peru Med Trop. 1993;7(1):61-5.

36. Cabrera R, Luna-Pineda MA, Suarez-Ognio L. Nuevo caso de infección humana por una larva de Pseudoterranova decipiens (Nematoda, Anisakidae) en el Perú. Rev Gastroenterol del Perú. 2003;23(3):217-20.

37. Takahashi S, Ishikura H, Kikuchi K. Anisakidosis: Global Point of View. In: Ishikura H, editor. Host Response to International Parasitic Zoonoses. Heidelberg: Springer; 1998. p. 112.

38. Yorimitsu N, Hiraoka A, Utsunomiya H, Imai Y, Tatsukawa H, Tazuya N, et al. Colonic intussusception caused by anisakiasis: A case report and review of the literature. Intern Med. 2013;52(2):223-6. DOI 10.2169/ internalmedicine.52.8629.

39. Shimamura Y, Muwanwella N, Chandran S, Kandel G, Marcon N, Shimamura Y, et al. Common Symptoms from an Uncommon Infection: Gastrointestinal Anisakiasis. Can J Gastroenterol Hepatol. 2016;2016:1-7. DOI 10.1155/2016/5176502. 
40. García García JM, Romero Arauzo MJ. Dolor torácico anginoso como manifestación inicial de anisakiasis gástrica. An. Med. Interna (Madrid). 2004;21 (4):185-6. DOI 10.4321/S0212-71992004000400008.

41. Mercado R, Torres P, Maira J. Human case of gastric infection by a fourth larval stage of Pseudoterranova decipiens (Nematoda, Anisakidae). Rev Saude Publica. 1997;31(2):178-81. DOI 10.1590/s003489101997000200012 .

42. Prester L. Seafood Allergy, Toxicity, and Intolerance: A Review. J Am Coll Nutr. 2016;35(3):271-83. DOI 10.1080/07315724.2015.1014120.

43. Cuéllar C, Daschner A, Valls A, De Frutos C, Fernández-Fígares $\nabla$, Anadón AM, et al. Ani s 1 and Ani s 7 recombinant allergens are able to differentiate distinct Anisakis simplex-associated alleroic clinical disorders. Arch Dermatol Res. 2012;304(4):283-8. DOI 10.1007/s00403-012-1206-8.

44. Martínez E, Loaiza L, Bastidas G. Anisakiosis. Comunidad y Salud. 2009;7(2):18-22.

45. Rodríguez-Mahillo AI, González-Muñoz M, Moneo I, Solas MT, Mendizábal A, de las Heras C, et al. Allergenic properties and cuticle microstructure of Anisakis simplex L3 after freezing and pepsin digestion. J Food Prot. 2008;71(12):2578-81. DOI 10.4315/0362-028X-71.12.2578.

46. Palomba M, Paoletti M, Colantoni A, Rughetti A, Nascetti G, Mattiucci S. Gene expression profiles of antigenic proteins of third stage larvae of the zoonotic nematode Anisakis pegreffii in response to temperature conditions. Parasite. 2019;26(52):1-12. DOI 10.1051/parasite/2019055.

47. Serrano-Moliner M, Morales-Suarez-Varela M, Valero MA. Epidemiology and management of foodborne nematodiasis in the European Union, systematic review 2000-2016. Pathog Glob Health. 2018;112(5):24958. DOI 10.1080/20477724.2018.1487663.

48. Del Rey-Moreno A, Valero-López A, Lozano-Maldonado J, Gómez-Pozo B, Mayorga-Mayorga C, TorresJaén MJ, et al. Use of anamnesis and immunological techniques in the diagnosis of anisakidosis in patients with acute abdominal pain. Rev Esp Enfermedades Dig. 2008;100(3):146-52. DOI 10.4321/S113001082008000300004 .

49. Castellanos JA, Santana-piñeros AM, Mercado R, Peña S, Pustorrh C, Cruz-Quintana Y. Presence of anisakid larvae in commercial fishes landed in the
Pacific coast of Ecuador and Colombia. Infectio. 2018;22(4):206-12. DOI 10.22354/in.v22i4.739.

50. Ulloa Ulloa LA. Determinacion de parásitos en pescados mas comercializados en los mercados 10 de noviembre y 24 de mayo (Bellavista), ubicado en el canton Guaranda, provincia Bolivar [Trabajo de grado Doctor en Medicina Veterinaria y Zootecnia]. Ecuadro:Universidad Estatal de Bolivar; 2008.

51. Olivero J, Baldiris R, Olivero Verbel J, Baldiris Avila R. Parásitos en peces colombianos: Están enfermando nuestros ecosistemas [internet]. [Consutado $2018 \mathrm{sep}$ 3].Disponible en: https://cutt.ly/feBW9NO

52. Ruiz L, Vallejo A. Parámetros de infección por nemátodos de la familia Anisakidae que parasitan la Lisa (Mugil incilis) en la bahia de Cartagena (Caribe Colombiano). Rev Intropica. 2013;8:53-60.

53. Wadnipar Cano LM. Evaluación de la infección parasitaria por nemátodos anisákidos en peces de interés comercial en el municipio de San Marcos (Sucre) [Trabajo de grado Maestría en Desarrollo Sostenible y Medio Ambiente]. Manizales: Universidad de Manizales; 2013.

54. Pardo S, Mejía PK, Navarro VY, Atencio GV. Prevalencia y abundancia de Contracaecum sp. en rubio (SaIminus affinis) en el río Sinú y San Jorge: Descripción morfológica. Rev MVZ Córdoba. 2007;12(1):887-96. DOI 10.21897/rmvz.433.

55. Oliviero Verbel J, Baldiris Avila R, Caballeor Gallardo K, Pacheco Londoño Y. Nemátodos de la Familia Anisakidae en Especies Ícticas de la Bahía de Cartagena y el Canal del Dique. In: Parásitos en peces colombianos: están enfermando nuestros ecosistemas [internet]. [Consutado 2018 sep 3]. Dispobible en: https:// cutt.1y/AeBEO83

56. Oliviero Verbel J, Barrios Puerta M, Baldiris Avila R. Merluzas (Merluccius gayi) Parasitadas Adquiridas en Establecimientos Comerciales de Cartagena y Barranquilla. In: Olivero-Verbel J, editor. Parásitos en peces colombianos: Están enfermando nuestros ecosistemas. Editoral U. Cartagena: Editorial Universidad de Cartagena; 2008. p. 85-101.

57. Patiño J, Olivera M. Anisakiasis gastro-aléroica, primera descripción de un caso en Colombia y revisión de literatura. Biomédica. 2019;39(2):1-8. DOI 10.7705/ biomedica. $\nabla 39 i 2.3936$.

58. Puccio F, Cifarelli D, Blanco F, López E, Sarmiento L, Ordaz R, et al. Reactividad aléroica a Anisakis 
simplex y su asociación con asma bronquial en niños escolares del estado Nueva Esparta, Venezuela. Bol. malariol. salud ambient. 2008;(2):145-2.

59. Figueiredo I, Vericimo M, Terra L, Ferreira T, Sao Clemente SC, Teixeira G. Association between immunoreactivity to Anisakis spp. antigens and high-risk pregnancy. Acta Parasitol. 2015 Dec;60(4):609-13. DOI 10.1515/ap-2015-0085.

60. Castellanos Garzón JA. Nematodos anisákidos en peces de consumo y prevalencia de anticuerpos antiAnisakis en una población de Colombia. Cali: Universidad del Valle; 2018. 